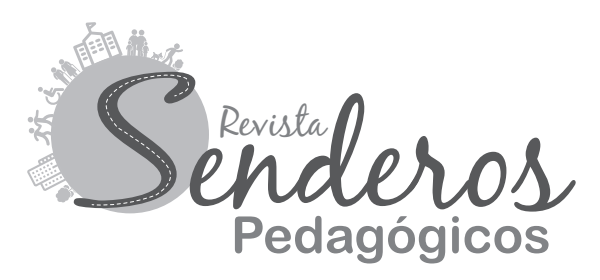

\title{
Las TIC como mediadoras del aprendizaje en niños y niñas con necesidades educativas especiales en los grados de transición y primero ${ }^{1}$
}

\author{
ICT as mediators of learning in children with special needs \\ in the transitional and first grades
}

\author{
Autoras: \\ Maria Camila López Bedoya ${ }^{2}$ \\ Nancy Milena Yotagrí Moreno ${ }^{3}$ \\ Recibido: 28/02/19 \\ Aprobado: 27/11/19
}

1. La investigación realizada durante un año como requisito de graduación en la Licenciatura en Educación Preescolar.

2. Licenciada en Educación Preescolar. Tecnológico de Antioquia Institución Universitaria. mariaclobe7@hotmail.com

3. Licenciada en Educación Preescolar. Tecnológico de Antioquia Institución Universitaria.

nancymilenay@hotmail.com

\section{Resumen}

La incorporación de las Tecnologías para la Información y la Comunicación (TIC) en el sistema educativo se convierte en un reto contemporáneo, en cuanto a la innovación y el impulso de la creatividad de los maestros, en los aprendizajes y desarrollo de habilidades y destrezas de todos los estudiantes y, de manera particular, en estudiantes con Necesidades Educativa Especiales (NEE); en este sentido, la investigación se orienta a analizar los aportes de las TIC y el Diseño universal para el aprendizaje (DUA) con la intención de favorecer el aprendizaje en niñas y niños con NEE en los grados de transición y primero, y proponer estrategias que puedan ser aplicadas en escenarios educativos.

La investigación se fundamenta en el paradigma cualitativo con utilización del enfoque interpretativo, y de las técnicas de análisis documental y entrevista, los resultados señalan que es de baja incorporación en la enseñanza de las 
TIC y el DUA, hallazgo que muestra la necesidad de relacionar en los currículos tecnología accesible con enseñanza en procesos de inclusión educativa para favorecer el aprendizaje de todos los estudiantes.

Palabras clave: Necesidades Educativas Especiales (NEE), Tecnologías de la Información y la Comunicación (TIC), Diseño Universal del Aprendizaje (DUA).

\begin{abstract}
The incorporation of Information and Communication Technologies (ICT) in the education system becomes a contemporary challenge, in terms of innovation and the promotion of the creativity of teachers and in the learning and development of skills and abilities of all students and particularly in students with Special Educational Needs (NEE); In this sense, the research aims to
\end{abstract}

analyze the contributions of ICT and Universal Design for Learning (ULD) for the promotion of learning in girls and boys with SEN in the transition and first grades, and propose strategies that can be applied in educational scenarios.

The research is based on the qualitative paradigm with the use of the interpretive approach, using the techniques of documentary analysis and interviewing, the results indicate that ICT and ULD are of low incorporation in teaching, a finding that shows the need to relate in the curricula accessible technology with teaching in processes of educational inclusion to favor the learning of all students.

Keywords: Special Educational Needs (SEN), Information and Communication Technology (ICT) and Universal Learning Design (ULD).

\section{Introducción}

La Organización para la Cooperación y el Desarrollo Económico (OCDE), en su informe Panorama educativo: indicadores de la OCDE edición 2003, señalan que el nivel de competencias y uso de recursos educativos digitales por parte de los docentes es bajo. Parece común observar a los docentes cumpliendo un rol más de usuarios y consumidores de contenidos curriculares que productores y diseñadores de los mismos, en este sentido autores como (Balanskat \& Blamire, 2006; Area , 2008; Coll 2008), establecen que a pesar del aumento en incorporación de las TIC en las escuelas, las didácticas utilizadas por los docentes siguen siendo las mismas, es decir, los enfoques tradicionales de enseñanza siguen abanderando las metodologías y prácticas de la comunidad docente, (Pelgrum, 2000), indica que la mayoría de los profesores no transforma sustancialmente su práctica docente al integrar tecnología en el aula, lo que hace es acomodar la tecnología a su práctica actual. Es decir, existen obstáculos a la integración de las TIC en los procesos de la enseñanza y el aprendizaje, citado en Carneiro, Toscano, \& Díaz (2009, p.42). 
Adicional, en Colombia existen políticas de atención inclusiva para la población en situación de vulnerabilidad, es posible evidenciar múltiples dificultades para garantizar la participación efectiva en el sistema educativo de manera particular de la población con NEE, como las actitudes por parte de los docentes, la falta de ajustes razonables y flexibilización en el currículo, además de la ausencia de metodologías y didácticas diversas. La falta de apoyo específico desde las TIC, se convierten en barreras que impiden la participación efectiva de esta población en los procesos de enseñanza aprendizaje.

Para dar respuesta a esta necesidad, se orientó la investigación con el propósito de "analizar los aportes de las TIC y el DUA para el favorecimiento del aprendizaje en niñas y niños con NEE en los grados de transición y primero”, proyectando los objetivos específicos a la caracterización de investigaciones que aplican las TIC y DUA a niños y niñas con NEE y relacionar los aportes de estas con el aprendizaje para estos los niños y niñas, se orienta la investigación mediante el enfoque cualitativo, ya que el objeto de estudio es observable, se puede indagar y existe en la interacción con el contexto, el método es de tipo exploratorio, porque brinda un primer acercamiento al problema que se pretende conocer y dar respuesta, por medio del diseño de estrategias para el aprendizaje de los estudiantes. Con este tipo de investigación se logra obtener la información inicial para dar continuidad a una investigación posterior, centrada en el diseño de estrategias para favorecer el aprendizaje de los estudiantes con NEE que articule las TIC en la enseñanza en la educación preescolar.

Se utiliza la técnica del análisis documental y para recolectar la información se utiliza una ficha de análisis de contenido, donde se describen los siguientes campos: Referencia bibliográfica, resumen, palabras clave, conclusión, vacíos y aportes que suscitan de las investigaciones, artículos y estudios de caso, los cuales son base para el desarrollo adecuado del análisis, para dar respuesta a los objetivos planteados.

En el plan de análisis, se definen los siguientes pasos:

Análisis documental:

Los trozos de contenido de las investigaciones revisadas se incorporan en una hoja de cálculo, se sigue con el filtro de unidades de sentido, para establecerle códigos abiertos y luego pre categorizar y categorizar, para el análisis, se agrupan los códigos abiertos en cada categoría, definiendo el porcentaje sobre la totalidad de códigos obtenidos, el cual conllevo a obtener frecuencias y analizar más repetitivas y las de poca proporción para así realizar el análisis de las tendencias llevándolas a los resultados agrupadas en categorías. 


\section{Marco de referencia conceptual}

El enfoque de la educación inclusiva ha promovido la posibilidad de acoger en la institución educativa a todos los estudiantes, con características personales, culturales o con diferentes formas de aprender para atenderlos de manera pertinente y con calidad. Se parte de la premisa de que todos los estudiantes pueden aprender, siempre y cuando su entorno educativo ofrezca condiciones de accesibilidad para todos y provea experiencias de aprendizaje significativas para todos los actores que allí se encuentran. Significa que todos los niños, niñas y jóvenes de una comunidad determinada puedan estudiar juntos, expresa Correa "le corresponde a la educación aportar para romper este círculo de circunstancias, de manera que ellos encuentren alternativas para salir de su situación de vulnerabilidad y segregación” (2017, p. 147).

Promover oportunidades de aprendizajes a lo largo de la vida en los estudiantes y potenciar su actuación en cualquier ámbito de la vida personal, social, cultural, requiere de una postura desde la perspectiva del desarrollo humano, que reconozca los sujetos como seres integrales, con capacidades, habilidades y actitudes, capaces de actuar de forma coherente con los contextos socioculturales y con las características individuales, esta postura elimina la mirada que se tiene de los estudiantes con NEE, como “incapaces”, poniéndose en dialogo con el enfoque del desarrollo humano.

En la investigación se asumen las Necesidades Educativas Especiales, como aquellas necesidades educativas individuales que no pueden ser resueltas a través de los medios y los recursos metodológicos que habitualmente utiliza el docente para responder a las diferencias individuales de sus estudiantes y que requieren para ser atendidas de ajustes, recursos o medidas pedagógicas especiales o de carácter extraordinario, distintas a las que requieren comúnmente la mayoría de los estudiantes (MEN, 2006, p.31).

Por su parte, el Diseño Universal del Aprendizaje (DUA), se entiende como el diseño de productos, entornos, programas y servicios que puedan utilizar todas las personas, en la mayor medida posible, sin necesidad de adaptación ni diseño especializado. (MEN, 2017, p.5). Es un acercamiento que permite la eliminación de barreras, la formación de aprendices expertos, la creación de currículos flexibles. Complementa esta definición Restrepo:

El diseño universal del aprendizaje (DUA) en la educación es un tema que en los últimos años ha sido eje de gran interés para el campo educativo: las construcciones socioeducativas en relación con la diversidad, la 
diferencia, la inclusión, la exclusión, la flexibilización de los currículos y el mejoramiento de los procesos de enseñanzaaprendizaje asientan una variedad de perspectivas que constituyen un reto significativo para los sistemas educativos. (2018, p. 40)

Por otro lado, las TIC, según Gil (2002), constituyen un conjunto de aplicaciones, sistemas, herramientas, técnicas y metodologías asociadas a la digitalización de señales analógicas, sonidos, textos e imágenes, manejables en tiempo real. Por su parte, Ochoa y Cordero (2002), establecen que son un conjunto de procesos y productos derivados de las nuevas herramientas (hardware y software), soportes y canales de comunicación, relacionados con el almacenamiento, procesamiento y la transmisión digitalizada de la información.

Así mismo, Thompson y Strickland, (2004) definen las tecnologías de la información y comunicación, como aquellos dispositivos, herramientas, equipos y componentes electrónicos, capaces de manipular información que soportan el desarrollo y crecimiento económico de cualquier organización. Cabe destacar que en ambientes tan complejos como los que deben enfrentar hoy en día las organizaciones, sólo aquellos que utilicen todos los medios a su alcance, y aprendan a aprovechar las oportunidades del mercado visualizando siempre las amenazas, podrán lograr el objetivo de ser exitosas.

\section{Resultados}

Para llegar a los resultados, se establece una escala de valoración en tres niveles de importancia, establecida a partir de la frecuencia encontrada en las TIC y el DUA, la información analizada en las fichas, se procesa en Excel, en el proceso de agrupación, códigos abiertos, luego pre categorías y por ultimo las categorías, en estas últimas se registran las frecuencias de los códigos, en las cuales se retoma el filtro más alto y el más bajo, se divide por tres, realizando una repartición proporcional a las significaciones alta, media y baja.

\section{Resultados investigaciones en las TIC}

Se analizaron 15 documentos con resultados de investigaciones en el uso de las TIC en la educación, la frecuencia más alta se registra en 25 y la más baja en 1, lo que permite originar las tres escalas dan como resultado 11 categorías, de las cuales 3 se encuentran en una significación alta, 4 categorías en una significación media y 5 categorías en una significación baja, según la escala que se propuso para la interpretación, como se aprecia en el siguiente cuadro: 
Tabla $\mathbf{N}^{\circ}$ 1. Escala de valoración en resultado de las TIC.

\begin{tabular}{|c|c|}
\hline Escala de valoración & Rango de frecuencia \\
\hline Significación alta & $25-16$ \\
\hline Significación media & $15-8$ \\
\hline Significación baja & $7-1$ \\
\hline
\end{tabular}

Fuente elaboración propia.

Tabla $\mathbf{N}^{\circ}$ 2. Clasificación de las categorías en la escala de valoración.

\begin{tabular}{|c|c|}
\hline Categorias & Escala de valoración \\
\hline Tecnología accesible & Significación alta \\
\hline Inclusión educativa & Significación alta \\
\hline Aprendizaje & Significación alta \\
\hline Didáctica & Significación media \\
\hline Recursos & Significación media \\
\hline Convivencia & Significación media \\
\hline Comunicación & Significación media \\
\hline Contexto educativo & Significación baja \\
\hline Compromiso & Significación baja \\
\hline Currículo & Significación baja \\
\hline Formación & Significación baja \\
\hline
\end{tabular}

Fuente elaboración propia.

\section{Categorías de significación alta}

- Categoría Tecnología accesible: esta se explica en las subcategorías finalidad educativa, recursos, finalidad de la educación y estrategias de enseñanza. La finalidad educativa apunta a una atención individualizada, donde las TIC fortalecen la disposición de los estudiantes para progresar en torno a las discapacidades, modificando la vida de las personas, la ruptura de limitaciones físicas; siendo un factor positivo para combatir el aislamiento e incrementando así los procesos de comunicación e intercambio de saberes, facilitando la inclusión social, favoreciendo el desarrollo de las funciones cognitivas, que puedan ser utilizadas en otros contextos así como la autonomía y la participación, donde los 
Las TIC como mediadoras del aprendizaje en niños y niñas con necesidades educativas especiales en los grados de transición y primero

estudiantes puedan mejorar su habilidad mental, reconocer, apoyar y estimular sus habilidades, rompiendo barreras temporales y espaciales, donde se pueden ofrecer cambios cualitativos y cuantitativos. En cuanto a la subcategoría recursos, el uso de las TIC en edad temprana favorece de manera individual a la niña o al niño, por otro lado, los recursos se deben incorporar las TIC al currículo. En la subcategoría finalidad de la educación se encuentra que utilizar las TIC se puede convertir en un efecto novedoso, aumentando la motivación en los niños y niñas a la hora de aprender. También se hace referencia a la subcategoría estrategias de enseñanza, en esta se apunta a la simulación, la cual proporciona una situación artificialmente real, cuyo objetivo se dirige a que el alumno logre un aprendizaje, a la par que le ofrece información a uno o varios sentidos, ᄀvisión, sonido, tacto y gusto- para que de esta forma se sienta inmerso en un mundo que reacciona ante sus acciones.

- Categoría Inclusión Educativa: se encontraron las siguientes subcategorías adaptación, finalidad educativa y flexibilidad educativa. La adaptación respecto a las TIC apunta a adecuar las necesidades o demandas de cada persona; por ejemplo, en las formas de aprender, se promueven procesos de enseñanza aprendizaje centrados en los estudiantes, se plantea una flexibilidad curricular ya que la educación regular debe estar en capacidad de incrementar sus estrategias metodológicas para promover la accesibilidad de todos los estudiantes; el acceso de las TIC en la educación, es inherente en el reconocimiento de la igualdad de los estudiantes.

Por otro lado, se encuentra la sub categoría finalidad educativa, las TIC ayudan a los cambios de las escuelas y entornos, lo que la escuela proyecta es preparar a la gente para este entorno, si este cambia, la actividad de la escuela tiene que cambiar, mediante la participación de toda la comunidad, basada en el aprendizaje dialógico, las comunidades de aprendizaje generan éxito educativo, así como una mejora de la convivencia. La finalidad educativa apunta a la comunicación interpersonal en la que primen legítimas actitudes de respeto, empatía, solidaridad, valoración, y responsabilidad recíproca; por otra parte la finalidad educativa debe estar orientada al acceso a la información y la interacción con las diferentes dinámicas de las prácticas, desarrollando las competencias, capacidades y habilidades cognitivas de una manera integrada para extraer un mejor provecho de los aprendizajes, teniendo en cuenta que las TIC pueden mitigar problemas de los niños y niñas con discapacidades, al permitir compensar deficiencias serias de los órganos sensoriales y del aparato motor, ya que la tecnología es algo transversal y puede ser centrada en el déficit del alumno. 
La finalidad educativa rescata que el uso de las TIC puede potenciar actividades colaborativas y cooperativas entre los estudiantes, facilitando la adquisición de la competencia digital; por otro lado, proporciona la inclusión y la participación de las personas con algún tipo de dificultad en el proceso educativo. Hay que tener presente que se está generando un impacto en la creación de condiciones para la igualdad y el mejoramiento de la calidad de vida. Cabe rescatar que dependiendo de la forma como se implementen las TIC, pueden tener un impacto que facilite o restrinja su uso. Como finalidad educativa se apunta a la implementación de propuestas apoyadas por la tecnología; se pretende eliminar las limitaciones en el aprendizaje, logrando cambios en la manera de pensar y acceder al conocimiento, para ello se sugiere implementar el hardware como facilitador de intercambio de datos, con tecnologías adaptativas, teniendo un acceso a la información siendo estos un instrumento de inclusión.

Otra sub categoría es flexibilidad educativa, la cual sugiere que los programas deben ajustarse a criterios ergonómicos generales, como idoneidad del diseño, adaptación de contenidos a distintos niveles de dificultad, sistema de ayudas progresivas, evaluación de la ejecución, refuerzos adecuados al sistema perceptivo del sujeto. En la subcategoría formas de aprendizaje se deben tener en cuenta las particularidades de cada alumno, el ritmo de aprendizaje de los niños y niñas debe ser adecuado al aula, teniendo en cuenta que somos una sociedad plural con diferentes formas de pensar, aprender y actuar.

En la sub categoría recursos, el internet pone a disposición de las escuelas todo lo necesario para hacer posible las comunidades virtuales de aprendizaje intercultural, a través de multitud de herramientas para favorecer la comunicación, la construcción compartida de conocimientos y el intercambio de información acerca de experiencias en educación intercultural entre todos los miembros de las comunidades educativas.

- Categoría aprendizaje: se encontraron las subcategorías aplicación, aprendizaje visual, finalidad educativa, formas de aprender, logros, memoria, saberes. En la sub categoría aplicación es importante la incorporación de las TIC al currículo de la carrera docente; como contenido, eje transversal y uso de las TIC. Como contenido desde el inicio de la misma, puede ser como: curso propedéutico; asignatura particular; como eje transversal que impregne todo el currículo; con el uso de las TIC (e-mail, Chat, espacios, blog, foros de discusión, uso de ambientes basados en la Web, presentaciones, software educativos entre otros) usando efectivamente, el correo electrónico, el Chat, las presentaciones en PowerPoint y otras herramientas en formato electrónico en los 
cursos regulares, con el modelado y la posibilidad de transferencia en otros contextos y con contenido desde el inicio de la carrera a través de las TIC como cursos en línea, páginas web, entre otros.

En la sub categoría aprendizaje visual, se refiere a la modalidad de representación visual para las personas. La sub categoría finalidad educativa, apunta a nuevos métodos de enseñanza/aprendizajes constructivistas, que contemplan el uso de las TIC como instrumento cognitivo (aprender con las TIC) y para la realización de actividades interdisciplinarias y colaborativas. Es necesario que el alumno, apoyado y guiado por el profesor, sea capaz de "aprender a aprender"; esto implica acceder a la información, comprenderla, resaltar las ideas fundamentales, estructurarla, y tener una visión crítica sobre la misma, por lo tanto, el alumno pasa a ser el centro del proceso de aprendizaje, es el que construye el conocimiento, a través del apoyo y guía del profesor. Al respecto Correa, J., Suárez, J., Ramírez, P., Escobar, V. consideran que se debe “comprender la enseñanza como una acción que demanda reconocer las diferencias de los estudiantes, le advierte al maestro la importancia de organizar y planificar las acciones a desarrollar en el aula, para favorecer el aprendizaje de los estudiantes" (2019, p. 53).

En la sub categoría formas de aprender, la educación regular debe estar en capacidad de incrementar sus estrategias metodológicas, su flexibilidad curricular, promoviendo la accesibilidad de todos los estudiantes no solo de los que se encuentran en condición de discapacidad, promoviendo procesos de enseñanza aprendizaje centrados en todos los estudiantes. Mientras que en la sub categoría formas de aprendizaje, es de gran importancia a la hora de enseñar a los niños y niñas y para esto se debe tener en cuenta el ritmo y estilo de aprendizaje de cada uno para obtener un aprendizaje significativo.

Sub categoría memoria con esta se pretende fortalecer la estructuración y la memoria a largo plazo y en la sub categoría de saberes se pretende que los niños y niñas construyan sus propios conocimientos a partir de saberes previos. Para finalizar las subcategorías mencionadas, se traen a colación logros, los cuales estipulan mejorar los aprendizajes de los niños y niñas, donde el estudiante se vea favorecido en su auto aprendizaje.

\section{Categorías de significación media}

- Categoría didáctica: las subcategorías que aparecen en esta parte son adaptación y estrategias de enseñanza. En la sub categoría adaptación, las TIC adoptan un papel fundamental para acceder a la información, transformando los espacios de aprendizaje, para desarrollar nuevas 
fórmulas relacionales o proporcionar formatos múltiples y diversificados de acceso al conocimiento. En la sub categoría estrategias de enseñanza, el sistema facilita el desarrollo de la clase debido a que el concepto está organizado y categorizado, se observa que la manipulación de objetos con ayuda y las opciones pedagógicas y didácticas con apoyo en las TIC tienen mayor impacto en contraste con la educación tradicional, las TIC deben ser utilizadas como recursos de apoyo para el aprendizaje académico de las distintas materias curriculares, y para la adquisición y desarrollo de competencias específicas en TIC. Las estrategias de enseñanza deben ser innovadoras, generando estrategias didácticas y teniendo en cuenta que el docente debe ser consciente que debe existir una preparación de la clase. Las estrategias de enseñanza apuntan a la diversidad de formas de presentar los contenidos a las niñas y niños; buscan crear ambientes de aprendizaje facilitando oportunidades a los alumnos para que ellos construyan conceptos, desarrollen habilidades de pensamiento, valores y actitudes; la herramienta de las TIC permite a los niños y niñas aprender de forma más atractiva, amena, divertida y sencilla.

- Categoría recursos: donde se encontró la sub categoría recursos, la cual propone un acercamiento de materiales interactivos y al material disponible, por otro lado, se encuentra la motivación a los estudiantes en la construcción de conocimientos; el uso de las TIC se apoya en estrategias cooperativas, se hace especial énfasis en desarrollo de determinadas competencias, como la digital y la de aprender a aprender, se fomentan los valores inclusivos y se sientan las bases del apoyo mutuo. Los recursos sirven para desarrollar nuevas fórmulas relacionales o proporcionar formatos múltiples y diversificados de acceso al conocimiento; las TIC se deben percibir tanto desde el punto de vista del hardware (componente físico de los ordenadores: teclados, impresoras, monitores) como del software (componente lógico: programas informáticos, navegadores). Los recursos apuntan a la gestión de los mismos, acceso a la información y al conocimiento, la creación de herramientas informativas que permiten el acceso a datos académicos en tiempo real, con lo cual el estudiante puede ajustarse al desarrollo de la asignatura, sin importar la limitación física o cognitiva que presente. En cuanto al uso de las TIC como recurso, en edad temprana favorece de manera individual a la niña o al niño, pretendiendo una alfabetización digital, ya que es de gran importancia el uso de entornos y metodologías facilitadoras del aprendizaje implementando las TIC como mediadoras de la educación, por lo tanto, se debe aprovechar que los estudiantes son hoy más sensibles a un entorno digital. 
- Categoría convivencia: se encontraron las subcategorías finalidad educativa, interacción y participación. La finalidad educativa, la cual invita a los docentes no solo a que sean personas competentes digitalmente, por el contrario, se invita a que sean capaces de aplicar esos conocimientos en el proceso educativo que favorezcan la innovación a través del uso de las TIC en contextos educativos inclusivos. La finalidad educativa pretende la interacción sujeto-maquina adaptable a las características de los usuarios; se rescata que el uso de las TIC puede potenciar actividades colaborativas y cooperativas entre los estudiantes.

Subcategoría interacción, es más motivante trabajar juntos y aprender juntos, no solo se colocan en práctica conocimientos propios del área, sino que también se tienen en cuenta valores como el respeto y el buen trato. Otra de las subcategorías corresponde a la participación la cual apunta a que diferentes culturas se encuentren y convivan para compartir actividades de tipo educativo.

- Categoría comunicación: se encontró la sub categoría interacción, la cual proporciona el intercambio de roles, la interacción comunicativa entre docentes, estudiantes y las TIC. Una de las tecnologías que prometen una importante contribución en un futuro, es la basada en gestos, donde los usuarios pueden comunicarse por medio de los gestos y de la voz.

\section{Categorías de significación baja}

- Categoría Contexto Educativo: donde se encontró la sub categoría contexto, en este se analizan las realidades educativas donde los niños y niñas poseen características diversas, con diferentes discapacidades, pensamientos, etc.

- Categoría compromiso: se encontraron las subcategorías participación y reflexión. Se habla de participación ya que los miembros de la comunidad y agentes activos son de gran importancia en el proceso escolar y del acompañamiento de los niños y niñas; la reflexión en esta sub categoría es que los docentes reflexionen sobre su rol.

- Categoría Currículo: surge en bajo significación en frecuencia, sin embargo, es el dispositivo donde se articulan todas las propuestas pedagógicas con apoyo de las TIC y el DUA, en los trabajos revisados se trabajan, pero no están plasmadas en los currículos.

- Categoría formación: surge la sub categoría interés, la cual plantea que la falta de computadores y software en las aulas de clase puede limitar la disponibilidad de los profesores a las tecnologías, además, las 
instituciones brindan poco tiempo a los profesores para familiarizarse con las TIC.

\section{Resultados Diseño Universal del Aprendizaje}

Se analizaron 12 documentos que hablan sobre el DUA en la educación, 12 es la frecuencia más alta en la codificación y 1 la más baja, dando como resultado 10 categorías, de las cuales 4 se encuentran en una significación alta, 2 categorías en una significación media y 4 categorías en una significación baja, según la escala que se propuso para la interpretación, la cual se presenta a continuación:

Tabla $\mathbf{N}^{\circ}$ 3. Escala de valoración en resultado del DUA.

\begin{tabular}{|c|c|}
\hline Grado de significación & Rango de frecuencias \\
\hline Significación alta & $12-8$ \\
\hline Significación media & $7-4$ \\
\hline Significación baja & $3-1$ \\
\hline
\end{tabular}

Fuente elaboración propia.

Tabla $\mathrm{N}^{\circ}$.

\begin{tabular}{|c|c|}
\hline Categorias & Escala de valoración \\
\hline Tecnología accesible & Significación alta \\
\hline Inclusión educativa & Significación alta \\
\hline Currículo & Significación alta \\
\hline Didáctica & Significación alta \\
\hline Recursos & Significación media \\
\hline Aprendizaje & Significación media \\
\hline Permanencia educativa & Significación baja \\
\hline Compromiso & Significación baja \\
\hline Participación & Significación baja \\
\hline Formación & Significación baja \\
\hline
\end{tabular}

Fuente elaboración propia. 
Las TIC como mediadoras del aprendizaje en niños y niñas con necesidades educativas especiales

en los grados de transición y primero

\section{Significación alta}

- Categoría Inclusión Educativa en la cual se encuentran varias subcategorías como flexibilidad educativa, principios, logros y disposición. En flexibilidad educativa nos dice que el DUA propone aplicar los tres principios en el proceso de diseño del currículo educativo, la planificación flexible y accesible, así mismo como la distribución del tiempo en el aula ya que este permite enseñar a todos de una manera personalizada y que cada alumno puede entender el contenido obteniendo, minimizando de esta manera las barreras que inciden en las transiciones y garanticen una adecuada calidad estableciendo horarios y objetivos para cada actividad a desarrollar, teniendo una adecuada organización para proveer y organizar adecuadamente los recursos humanos y materiales de apoyo que aseguran un adecuado aprendizaje.

Por otro lado, tenemos los principios, los cuales plantean que se debe favorecer el derecho a la educación para así aprender juntos, logrando minimizar las barreras que inciden en las transiciones y garantizando una educación de calidad, estableciendo objetivos, horarios y actividades propicias al aprendizaje. Así mismo en los logros se plantea que se debe favorecer el aprendizaje globalizado, mejorando de esta manera los resultados con los medios tecnológicos que con los materiales tradicionales.

Por última en esta pre categoría se encontró la disposición el cual plantea un enfoque de intervención que va dirigida a las modalidades de apoyo para que las clases sean más amenas y sobre todo que cada uno de los estudiantes se sienta cómodo y con un aprendizaje más accesible.

- Categoría Currículo: hay dos subcategorías la primera está basada en los saberes los cuales plantean los diversos lenguajes con los que se debe planear una clase, teniendo en cuenta los contenidos didácticos que deben ir entrelazados para que los alumnos puedan tener un aprendizaje los cuales están planteados como la música, lectura, imágenes, también se debe pensar en lo que se necesita aprender y que sea de agrado para los infantes.

Como segunda subcategoría se tiene la flexibilidad educativa el cual plantea que todos los niños, niñas, jóvenes y adultos sin exclusión, a través del proceso educativos, accedan al conocimiento y compartan una base de aprendizajes común que los prepare para participar 
activamente en las estrategias curriculares las cuales estas deben ser adaptadas a las necesidades de los alumnos y a todos los elementos básicos, teniendo como objetivo compartir los principios de la inclusión y valorar la diversidad como oportunidad para el aprendizaje.

- Categoría didáctica: cuenta con diferentes subcategorías para la cual en métodos de enseñanza se refiere a todas las metodologías didácticas que se deben implementar en las aulas de clase, teniendo en cuenta el estilo de aprendizaje de los alumnos, por otro lado en la aplicación de saberes se refiere a todas las prácticas de enseñanza que se deben tener en cuenta a la hora de un aprendizaje ya que no solo se aprende en un aula cerrada sino que también se debe tener en cuenta las salidas a diferentes espacios educativos que ofrece la institución educativa, este va relacionado a la acción pedagógica en la cual se deben crear espacios para la enseñanza y aprendizaje teniendo en cuenta la estrategia de enseñanza las cuales son una guía para la enseñanza y para desarrollar actividades que propicien información de diferentes maneras en los contextos significativos para la educación.

Por otro lado se encuentran los principios pedagógicos se refiere a establecer planteamientos didácticos que reconozcan la diversidad de los discentes y promuevan estrategias en el proceso de enseñanza, en la disposición se hace necesario propiciar espacios, medios, mecanismos y metodologías que permitan superar los obstáculos de la inclusión, por consiguiente en el contexto plantean que las aulas deben estar enriquecidas de acciones pedagógicas que permitan a los educandos una mejor manera de codificar la información y obtener un aprendizaje significativo así mismo se pretende que en la flexibilidad educativa y en las estrategias de permanencia los docentes apliquen métodos basados en el manejo de los materiales como medios utilizados para presentar los conocimientos, también tengan en cuenta el componente emocional ya que este es crucial en el aprendizaje y sobretodo tener una programación flexible ya que todos los alumnos no aprenden de la misma manera.

\section{Significación media}

- Categoría Recursos: se encuentran las pre categorías recursos y contenidos los cuales en los recursos dan a conocer el soporte y material físico y dinámico que se deben introducir en las clases y en los contenidos se refieren a la transformación que está teniendo la educación al implementar las TIC como mediador y ayuda en el aprendizaje. 
- Categoría Aprendizaje: hace referencia que las estrategias de aprendizaje hace referencia a las diferentes formas de acceso que se tienen en un contenido o las diferentes formas con las que se plantea y se da a conocer la temática, en aprendizaje significativo, las tecnologías permiten el acceso y la producción del aprendizaje, permitiendo en este los estilos de aprendizaje ya que todos los alumnos aprenden de diferente manera y lo que hace la tecnología es implementar estrategias acordes para que todos tengan una manera diferentes de codificar una información para llegar al aprendizaje de un tema, permitiendo de esta manera que las estrategias de enseñanza y el contexto proporcionen estrategias significativas e intencionadas en un contexto en el cual se respete las diferencias de los niños y niñas.

- Categoría Permanencia Educativa: en esta las siguiente pre categorías capacitación, contexto, disposición, estrategias de permanencia y flexibilidad educativa. En capacitación se deben instruir a los docentes para que estos ayuden a los alumnos a dominar el aprendizaje en sí mismo, que ellos pueden convertirse en aprendices expertos de un tema dirigido con diferentes materiales. En el contexto se refiere que las condiciones que inciden en la permanencia y desempeño del aprendizaje de los alumnos, por lo tanto, las aulas de clase deben ser un espacio donde convivan diferentes etnias, pensamientos, actuaciones, gustos, ritmos de aprendizaje y dificultades para así llegar y tener en cuenta los estilos de aprendizaje y las clases sean más individualizadas dependiendo la necesidad de cada individuo. Por otro lado, en la disposición se debe adecuar el currículo y el docente contar con una amplia gama de herramientas para llevar a cabo su clase. En las estrategias de permanencia y la flexibilidad educativa se refieren a promover las estrategias y acciones para así contrarrestar la deserción escolar y que el estudiante tenga una adaptación en el currículo.

- Categoría Compromiso: se cuenta con tres subcategorías las cuales corresponden a la disposición, la estrategia de enseñanza y el contexto, en la primera hace referencia a los docentes, que estos sean mediadores y facilitadores del aprendizaje, que acompañen a los estudiantes en su cotidianidad, la segunda se refiere a que los estudiantes con discapacidad se enriquezcan en conocimientos y puedan ser partícipes del aprendizaje y por último se debe generar un clima colaborativo apoyando y centrado en el aprendizaje tanto de los alumnos como del docente. 
- Categoría Participación: se cuenta con la subcategoría vinculación y esta hace referencia a la implicación e implementación de que los alumnos sean entes activos a la hora de la enseñanza y aprendizaje para que puedan tener los mejores resultados en su vida académica.

- Categoría formación: en la cual se encuentran como subcategorías la capacitación, la disposición y la flexibilidad educativa en la cual en la capacitación se hace referencia a la elaboración de materiales, la preparación docente, la representación de los conocimientos, conceptos y habilidades para la planificación de las actividades que es un proceso sistémico y flexible en el que se organiza y anticipa los procesos de enseñanza aprendizaje y se comparten un aprendizaje en común para la participación activa, por lo tanto las decisiones deben ser el resultado de un proceso flexible.

En la subcategoría disposición y flexibilidad educativa se tiene en cuenta facilitar la gestión de información mediante el uso de las técnicas y herramientas tecnológicas para una adecuada implementación de la enseñanza, así mismo el currículo debe estar flexible para que tanto los niños, niñas, adultos accedan a un aprendizaje significativo para sus vidas.

\section{Resultados relación TIC y DUA}

Se procede a relacionar los aportes del DUA y de las TIC para el aprendizaje de los niños y niñas con NEE, se tienen en cuenta las categorías de análisis altas, medias y bajas que se tuvieron en los resultados, relacionado las complementarias en cada nivel de significación.

Tabla $N^{\circ}$ 5. Relación categorías TIC y DUA.

\begin{tabular}{|c|c|c|c|}
\hline $\begin{array}{l}\text { Escala de } \\
\text { valoración }\end{array}$ & Categorías TIC & Categorías DUA & $\begin{array}{c}\text { Relación categorías TIC- } \\
\text { DUA }\end{array}$ \\
\hline $\begin{array}{l}\text { Significación } \\
\text { alta }\end{array}$ & $\begin{array}{l}\text { - Tecnología accesible } \\
\text { - Inclusión educativa } \\
\text { - Aprendizaje }\end{array}$ & $\begin{array}{l}\text { - Inclusión } \\
\text { educativa } \\
\text { - Didáctica }\end{array}$ & $\begin{array}{l}\text { - Tecnología accesible-Di- } \\
\text { dáctica-DUA } \\
\text { - Aprendizaje-enseñanza- } \\
\text { • inclusión educativa }\end{array}$ \\
\hline $\begin{array}{l}\text { Significación } \\
\text { media }\end{array}$ & $\begin{array}{l}\text { - Didáctica } \\
\text { - Recursos } \\
\text { - Convivencia } \\
\text { - Comunicación }\end{array}$ & $\begin{array}{l}\text { - Aprendizaje } \\
\text { - Recursos }\end{array}$ & $\begin{array}{l}\text { - Recursos-didáctica } \\
\text { - Convivencia- actividades } \\
\text { colaborativas- } \\
\text { - Comunicación accesible }\end{array}$ \\
\hline
\end{tabular}


Las TIC como mediadoras del aprendizaje en niños y niñas con necesidades educativas especiales en los grados de transición y primero

\begin{tabular}{|c|c|c|c|}
\hline $\begin{array}{l}\text { Significación } \\
\text { baja }\end{array}$ & $\begin{array}{l}\text { - Contexto educativo } \\
\text { - Compromiso } \\
\text { - Currículo } \\
\text { - Formación }\end{array}$ & $\begin{array}{l}\text { - Permanencia } \\
\text { educativa } \\
\text { - Compromiso } \\
\text { - Participación } \\
\text { - Formación }\end{array}$ & $\begin{array}{l}\text { - Compromiso-intereses- } \\
\text { expectativas-motivación } \\
\text { - Contexto educativo- } \\
\text { formación } \\
\text { - Participación- } \\
\text { compromiso } \\
\text { - Permanencia educativa }\end{array}$ \\
\hline
\end{tabular}

Fuente elaboración propia.

Se establece la relación entre categorías de las TIC y el DUA con el propósito de analizar las estrategias de enseñanza que se requieren para el aprendizaje de los estudiantes con necesidades educativas.

En primer lugar, en la escala de valor alta se encuentran las siguientes categorías ya relacionadas entre sí, tecnología accesible y didáctica, aprendizaje y enseñanza y por último en esta escala esta la inclusión educativa.

- Relación tecnología accesible y didáctica: lo que nos plantean es que se debe tener en cuenta la accesibilidad a los recursos, utilizando las TIC en la población infantil como instrumento de la inclusión educativa, generando así competencias a desarrollar de manera integrada; también se generan los medios colectivos y rompen las barreras temporales y espaciales para tener un acceso en tiempo real, favoreciendo de esta manera los procesos educativos innovadores, orientando las estrategias de actividades articuladas al aula de clase, estableciendo planteamientos didácticos que reconozcan la diversidad de los estudiantes y promuevan las estrategias en los procesos de enseñanza, generando espacios, medios, mecanismos y metodologías que permitan superar los obstáculos que se presentan en clase, por lo tanto se debe tener en cuenta los materiales considerados estos como los medios para presentar los conocimientos y que el estudiante logre el aprendizaje el cual es un componente esencial para ellos.

- Relación aprendizaje y la enseñanza: para esto en relación con las TIC y el DUA, se plantea que los procesos de enseñanza posibilitan el desarrollo de las funciones cognitivas, la construcción de conocimientos propias a través de los saberes previos y la transferencia en otros contextos; el alumno es capaz de aprender a aprender, pasando a ser el centro en su proceso de aprendizaje y el docente pasa a ser una guía y apoyo, así el estudiante va favoreciendo su autoaprendizaje a sus particularidades, por otro lado, el docente cuando incorpora las TIC en su enseñanza para generar una ayuda y mejora en la concentración y motivación de sus estudiantes, con diferentes formas de pensar, 
aprender y actuar, motivando en los estudiantes el desarrollo de su inteligencia, mejorando las habilidades mentales y favoreciendo los ritmos y estilos de aprendizaje en la educación, proporcionando experiencias significativas e intencionadas, en la categoría inclusión educativa se plantea que las TIC ayudan a la evolución de las discapacidades de los estudiantes, teniendo presente los cambios de la escuela y el entorno, asumiendo la participación activa de toda la comunidad educativa, disfrutando una comunicación interpersonal e incrementando los procesos de comunicación, flexibilizando el currículo y reconociendo las igualdades de los estudiantes los cuales son una parte activa en el aprendizaje teniendo presente los ritmos y estilos de aprendizaje y así adecuando la diversidad del aula, generando una ruptura de las limitaciones físicas, proporcionando la inclusión y la participación de las personas con algún tipo de dificultad en el proceso educativo, teniendo una planificación flexible y accesible, distribuyendo el tiempo en el aula favoreciendo el derecho a la educación mejorando los resultados con los medios tecnológicos y personalizando los contenidos de aprendizaje globalizado.

Por otro lado, en la escala de valor media se encuentran las siguientes categorías recursos y didáctica, convivencia y actividades colaborativas, y comunicación y accesibilidad.

- Relación recursos y didáctica: hablan del acercamiento de los materiales interactivos para la motivación a los estudiantes en la construcción del conocimiento, facilitando el desarrollo de las clases, apoyando las estrategias cooperativas, transformando espacios de aprendizaje en diversos accesos al conocimiento afirmando el aprendizaje académico en los centros escolares preparando las clases de diferentes formas de presentar los contenidos, buscando ambientes que favorezcan el aprendizaje teniendo estrategias pedagógicas que sean de innovación y los docentes proporcionen estrategias didácticas más atractivas, amenas y divertidas, gestionando los recursos, el acceso a la información y al conocimiento transformando la gramática escolar, creando espacios de enseñanza aprendizaje con materiales digitales generando diferentes formatos para el desarrollo de las actividades estableciendo planteamientos didácticos que reconozcan la diversidad de los discentes y promuevan estrategias en el proceso de enseñanza dando respuestas flexibles en contextos educativos diversos.

- Relación convivencia y actividades colaborativas: se refiere al reconocimiento de las diferentes culturas presentes en el aula de clase, para esto se debe tener en cuenta las actividades educativas, que estas sean motivantes a la hora de trabajar juntos y aprender juntos, teniendo una relación estudiante profesor y 
no estudiante máquina, por lo tanto el docente debe tener un factor positivo para combatir el aislamiento colocando en práctica no solo los conocimientos sino también los valores, el respeto y el buen trato por los demás, para así tener una correlación mutua para el aprendizaje y la enseñanza, en esta categoría se refieren a la comunicación y a la accesibilidad, en la cual se habla de los roles, en este caso se da la interacción comunicativa entre los docentes y los estudiantes, en primer lugar, los docentes deben tener presente las dificultades de cada estudiante para así planear las clases de forma divertida y teniendo presente lo que los estudiantes requieren a la hora de aprender y que este sea significativo. En segundo lugar, el estudiante debe afianzar sus conocimientos y tener motivación para aprender cosas nuevas, así se genera una comunicación asertiva y tanto el estudiante como el docente logran un intercambio de saberes.

Para finalizar con la relación de las categorías, se encuentra en las de significación baja, las cuales son importantes para el proceso de aprendizaje de los estudiantes con necesidades educativas, compromiso, interés, motivación y expectativas; contexto educativo y formación, participación y compromiso y por último la permanencia educativa.

- Relación contexto educativo y formación: esta se refiere a la realidad educativa, donde los docentes requieren de tiempo para familiarizarse con las TIC y para elaborar materiales didácticos para sus clases, los docentes, representan los conocimientos, conceptos y habilidades para generar la inclusión de los niños y niñas en las escuelas; los docentes tienen un proceso de planificación entendido como un proceso sistémico y flexible en que se organiza y anticipa los procesos educativos de enseñanza y aprendizaje, facilitando la gestión de información mediante el uso de las técnicas pertinentes para un adecuado aprendizaje, por lo tanto las decisiones deben ser el resultado de un proceso de reflexión que debe tomarse para llevar a cabo en la práctica.

- Relación participación y compromiso: se hace referencia a los miembros de la comunidad y agentes activos reflexionando sobre el rol docente y que este tenga un rol de mediador para facilitar el aprendizaje, los docentes acompañan a los estudiantes en su cotidianidad y que los alumnos tienen una implicación de forma activa en sus procesos de aprendizaje, en permanencia educativa se debe promover estrategias y acciones para contrarrestar la deserción escolar teniendo adaptaciones para los estudiantes en el aula, generando espacios donde se concluya diversas etnias, pensamientos, actuaciones, gustos, ritmos de aprendizaje y dificultades para la adecuación del currículo ayudando a dominar el aprendizaje en sí mismo, en definitiva, convertirse en aprendices expertos. 
La comunidad son agentes activos y reflexionan sobre el rol docente implementando estrategias que facilitan el aprendizaje de los estudiantes, los docentes acompañan a los estudiantes en su cotidianidad y los estudiantes con discapacidad enriquecen su enseñanza y participan progresivamente en los procesos y objetivos del aprendizaje, teniendo un clima de colaboración y apoyo centrado en el aprendizaje y enseñanza en los alumnos y docente, respectivamente.

\section{Discusión}

Después de haber realizado un análisis riguroso en diferentes fuentes bibliográficas, una lectura crítica, consiente y reflexiva de la gran variedad de textos, artículos, investigaciones que aportan al proceso y conocimiento de las TIC y el DUA desde diversos ámbitos y perspectivas, al respecto Jaramillo considera que "la inclusión de las TIC en el aula hace parte de los contenidos académicos trascendentales para la vida para enfrentarse positivamente a elementos sociales que vienen evolucionando como el teletrabajo y la educación virtual” (2015, p.51). La invitación en este artículo es a implementar y como docentes ser conscientes de que las TIC están a la vanguardia, que son llamativas y novedosas para los niños y niñas, en este sentido Betancur plantea que "el uso educativo adecuado de las TIC tiene que ver con la conciencia del docente que sea capaz de darle sentido a la riqueza conceptual y metodológica que requiere la tecnología como componente de la educación" (2012, p.58).

Las TIC son abordadas desde diversos puntos de vista, no solo como la computadora, el celular, la televisión, el internet, la radio, el DVD, la memoria USB, etc., sino también como aquellas herramientas físicas que se convierten en mediadoras y motivantes para el aprendizaje de los estudiantes, De Vita, citando a Gil (2002) define "las TIC como un conjunto de aplicaciones, sistemas, herramientas, técnicas y metodologías asociadas a la digitalización de señales analógicas, sonidos, textos e imágenes, manejables en tiempo real”2008, p.78.

Respecto al DUA cabe resaltar la importancia que posee repensarse como docentes, de qué manera se está implementando en el aula, si se hace caso omiso a ello o aún se piensa que todos los niños y niñas son iguales, y tienen un mismo ritmo de aprendizaje, Giné y Font, 2007, como señalan estos autores, se trata de un sistema de apoyo que favorece la eliminación de barreras físicas, sensoriales, afectivas y cognitivas para el acceso, aprendizaje y la participación de los alumnos. Esta nueva concepción de la accesibilidad es entendida como una condición imprescindible para garantizar la igualdad de oportunidades en el aula, en esta mirada, Correa y Restrepo. 
Las TIC como mediadoras del aprendizaje en niños y niñas con necesidades educativas especiales

en los grados de transición y primero

Conciben que el diseño universal del aprendizaje se convierte en uno de los enfoques más prometedores para asegurar que todos los alumnos puedan acceder a los contenidos y objetivos del currículo ordinario. Este permite ajustes razonables en las metas, los métodos, los materiales y las evaluaciones pues su intencionalidad busca satisfacer las necesidades individuales (2018, p.185).

Para el Ministerio de Educación Nacional de Colombia (2017), hace referencia al Diseño Universal del Aprendizaje (DUA): como ese diseño de productos, entornos, programas y servicios que puedan utilizar todas las personas, en la mayor medida posible, sin necesidad de adaptación ni diseño especializado (p.5), agregan Correa y Restrepo.

Este enfoque trata en definitiva de eliminar las barreras que le impiden a los individuos y grupos acceder plenamente al aprendizaje y los servicios mediante la apuesta por un diseño curricular y metodológico que tenga en cuenta las características heterogéneas de los educandos y los grupos (2017, p.55)

Se sugiere tener una mente abierta, apasionada a las nuevas propuestas, con apertura al cambio, para contribuir con la transformación de la educación tradicional en una educación constructiva, donde tanto docentes como estudiantes vivencien y hagan real el aprendizaje, se motiven por descubrir y explorar mediante las diversas capacidades de los cada niño y niña, y así promover el desarrollo de habilidades humanas, UNESCO, 1998 "Los rápidos progresos de las tecnologías de la información y la comunicación modifican la forma de elaboración, adquisición y transmisión de conocimientos”.

\section{Conclusiones}

- En la revisión de las investigaciones que apliquen las TIC en los grados de transición y primero, son escasos los referentes a la enseñanza de niños y niñas con NEE.

- Los hallazgos evidencian que las aplicaciones de las TIC en educación son cada vez más accesibles para el aprendizaje de los estudiantes con necesidades educativas, permitiéndoles obtener mejores desempeños y logros en diferentes áreas del currículo.

- La aplicación del DUA con las TIC requiere de un currículo flexible y una actitud con apertura al cambio e innovación en las prácticas pedagógicas de las instituciones y de los docentes. 
- En el análisis realizado a la relación de las TIC y el DUA, se puede encontrar que las categorías de significación baja, son de gran importancia para la formulación de las estrategias de enseñanza conducentes al logro del aprendizaje en los estudiantes.

- Las TIC son mediadoras del aprendizaje y se articulan a la enseñanza de los maestros, las cuales se deben utilizar de manera intencionada en el aprendizaje situado, estableciendo relaciones con situaciones de la vida diaria de los niños y niñas con necesidades educativas.

\section{Referencias}

Betancur, P. (2012). Tic en Educación ¿problema solución o posibilidad? Senderos Pedagógicos, (3), 39-49.

Carneiro, R., Toscano, J. C., \& Díaz, T. (2009). Los desafíos de las TIC para el cambio educativo. España: OEI.

Correa, J., Suárez, J., Ramírez, P., Escobar, B. (2019). Diversidad y educación. Saberes producidos en la Maestría en Educación del Tecnológico de Antioquia. Medellín: Publicar - T.

Correa, J., Restrepo. N. (2018). Atención a la diversidad retos y desafíos en la educación superior. En: Trends and challenges in higher in education in Latin America. Barcelona: Adaya Press.

Correa, J. (2018). A avaliação da aprendizagem no contexto da justiça educativa para população com deficiência na educação superior. Revista Brasileira de Educación Especial. (2), 89-102.

Correa, J., Restrepo. N. (2018). Modelo para la atención a la diversidad en el Tecnológico de Antioquia centrado en la justicia educativa y la equiparación de oportunidades. Medellín: Publicar - T.

Correa, J. (2017). Historicidad de procesos de exclusión y discriminación para grupos minoritarios en educación superior. Senderos Pedagógicos, (8), 141-153.

De Vita, N. (2008). Tecnologías de información y comunicación para las organizaciones del siglo XXI. Centro de Investigación de Ciencias Administrativas y Gerenciales. CICAG, (5), 1, 77-86.

Jaramillo, H. (2015). Las TIC: Un edublog como alternativa mejoradora en competencias de comprensión y producción textual. Senderos Pedagógicos (6), $41-51$ 
Ministerio de Educación, Cultura y Deporte. (2012). Educación Inclusiva. Iguales en la diversidad. Recuperado de http://www.ite.educacion.es/ formacion/materiales/126/cd/pdf/m3_ei.pdf

Ministerio de Educación Nacional (2006). Guía No.12 Fundamentación conceptual para la atención en el servicio educativo a estudiantes con Necesidades Educativas Especiales -NEE-. Recuperado de http:/www. colombiaaprende.edu.co/html/mediateca/1607/articles-75156_archivo.pdf

Ocde. (2003). Panorama educativo: indicadores de la OCDE edición 2003. Recuperado de http://www.oecd.org/education/skills-beyondschool/29881539.pdf

Ochoa, X. y Cordero, S. (2002). Las Nuevas Tecnologías de la Información y la Comunicación. Disponible en: http://www.ruv.itesm.mx/especiales/citela/ documentos/material/módulos/módulos2/contenidoii.htm

Restrepo, N. (2018). Diseño universal en la educación infantil. Reflexiones frente a su implementación e implicación para el proceso de enseñanzaaprendizaje. Senderos Pedagógicos, (9), 39-56.

Thompson, A. y Strickland, A. (2004). Administración Estratégica. México: Mc Graw Hill.

Unesco (18 al 22 noviembre de 1996) La Educación Superior En El Siglo XXI: Visión de América Latina y del Caribe. Ediciones CRESALC/UNESCO. Tomo 1 Habana Cuba. Recuperado de http://www.redalyc.org/pdf/761/76102311.pdf 


\section{De tal manera}

que la educación digital constituye una prioridad en la sociedad del conocimiento o digitalizada e interconectada

de múltiples modos de comunicación.

(Castells, 2000).

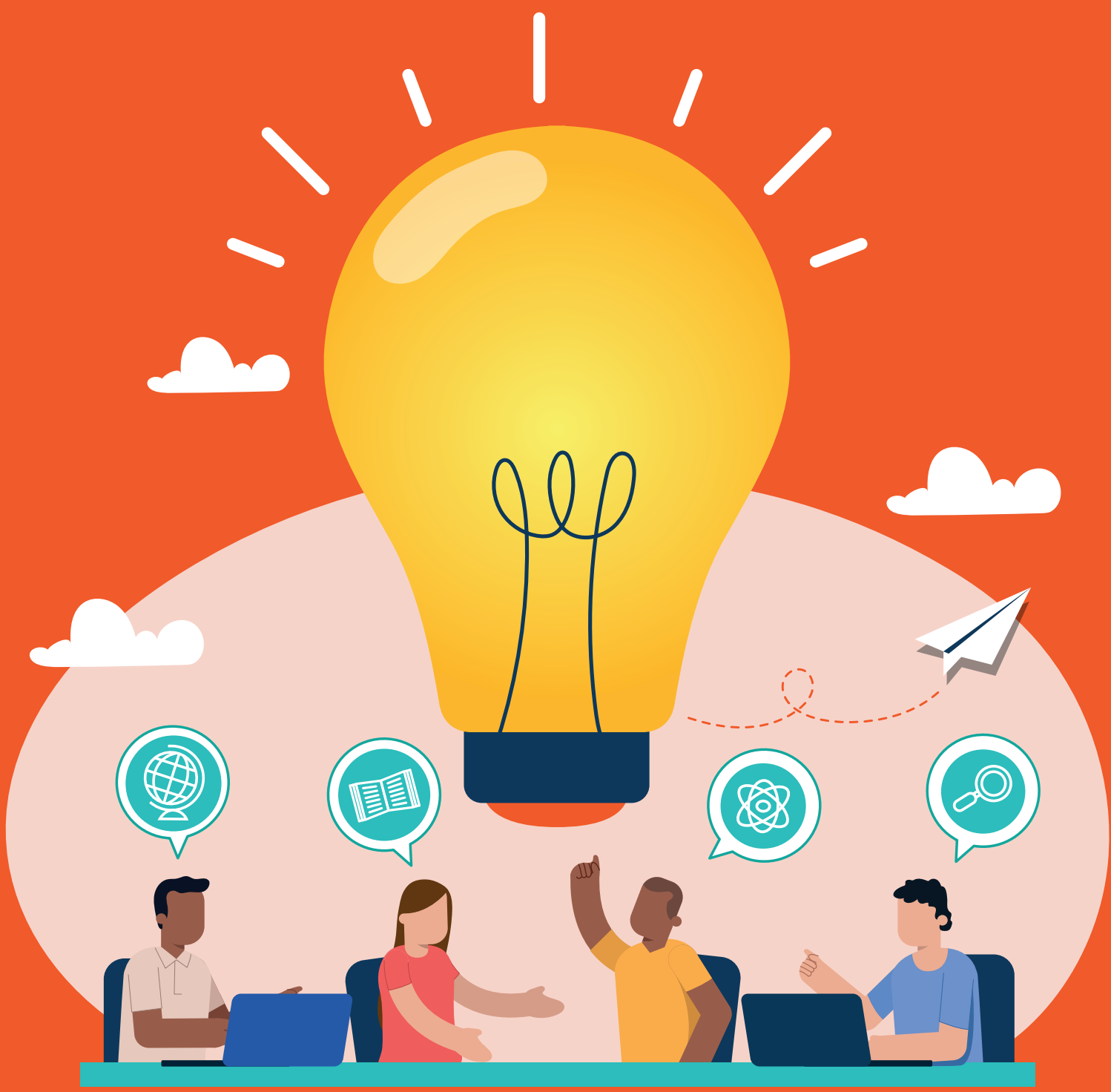

